\title{
Algorithm of oscillometric blood pressure measurement applied on microcontroller
}

\author{
$1^{\text {st }}$ Dávid Angyal \\ Department of Computer Science \\ Faculty of Informatics \\ University of Debrecen \\ Debrecen, Hungary \\ angyal.david@inf.unideb.hu
}

\author{
$2^{\text {nd }}$ Anikó Vágner \\ Department of Information Technology \\ Faculty of Informatics \\ University of Debrecen \\ Debrecen, Hungary \\ vagner.aniko@inf.unideb.hu
}

\author{
$3^{\text {rd }}$ István Juhász \\ Department of Information Technology \\ Faculty of Informatics \\ University of Debrecen \\ Debrecen, Hungary \\ juhasz.istvan@inf.unideb.hu
}

\begin{abstract}
Blood pressure measurement is a very common technique to monitor the condition of a patient to perform clinical studies of certain illnesses or classify the blood hypertension. On the market a number of blood pressure measurement devices are available, amoung them the oscillometric devices can be easily used under home circumstances.

Our oscillometric blood pressure measurement algorithm was implemented for a microcontroller environment. Our goal is to measure the blood pressure periodically during a 24-hour interval, store the records and transmit them to an application running on a $\mathrm{PC}$, which visualizes the records and make it possible to interactivelly examine the records.

In this article we introduce the implementation of the main steps of our oscillometric blood pressure algorithm with their difficulties.
\end{abstract}

Index Terms-automated blood pressure measurement, microcontroller, biometrical signal processing

\section{INTRODUCTION}

Nowadays blood pressure measurements serve as a conventional technique for physicians to gain information on patients' condition. Noninvasive blood pressure measurements are mainly used because invasive methods can be done only in clinical circumstances. The two most common used noninvasive methods are the auscultatory and the oscillometric method. Physicians prefer the auscultatory method because it is more accurate than the oscillometric method. The estimated error of the auscultatory method is at most $10 \mathrm{mmHg}$ compared to the invasive method. However, devices using oscillometric methods are most often used under home circumstances because people can use those devices very easily. There are many oscillometric devices available on the market. [4]

In this paper we introduce our oscillometric algorithm for microcontroller. Its main task is to perform blood pressure measurements periodically during a 24-hour interval and transmit the records to the BP Service application [13] of Cardiospy System [5] running on PC-side. One of goals of the microcontroller equipped with our algorithm is to transmit the information through the internet in order that further examination can be performed with the BP service application even during the examined 24-hour interval. This article focuses on the oscillometric algorithm.
On the PC-side the BP Service application equipped with interactive feautres can visualize all the recordings, additionally the users can change parameters in it so physicians and medical scientists can use it for the clinical studies of certain illnesses, blood hypertension classification, etc. Furthermore the BP service application is a part of the Cardiospy system which can process not only oscillometic blood pressure recordings but it supports many examinations taken by cardiologists. [13] [5]

Despite the fact that the recordings will be examined in the PC-side application the microcontroller's important task is to perform real-time blood pressure measurements and display the result to the patient. The results produced by the microcontroller serve as informative results to patients, but physicians may need more accurate results than the microcontroller can produce since the $\mathrm{PC}$-side application can also visualize the recordings and can change the parameters of the algorithm.

To sum up, our oscillometric algorithm works on a microcontroller device which measures blood pressure, displays informative results to patients and transmits the blood pressure measurement records to a PC-side application through the internet. But our goal was also that the device could work standalone and could be sold on the market without its PCside application.

\section{THE OSCILLOMETRIC METHOD}

In the case of oscillometric method, the cuff has a built-in sensor which perceives the pressure of the artery and the cuff. After cleaning away the pressure values made by the deflation of the cuff, we get an oscillation waveform, the oscillogram. The oscillometric method calculates the systolic pressure (SP), the diastolic pressure (DP) and the mean arterial pressure (MAP) based on the changes of the pressure in the oscillogram [2], [14], [7].Figure 1 shows an example of the cuff pressure curve and the corresponding oscillometric waveform.

The values of blood pressure can be derived from the cuff pressure curve at a given point. The method determines the point of the systolic pressure, the diastolic pressure and the mean arterial pressure. The point of the mean arterial pressure is the maximum point of the oscillogram. 


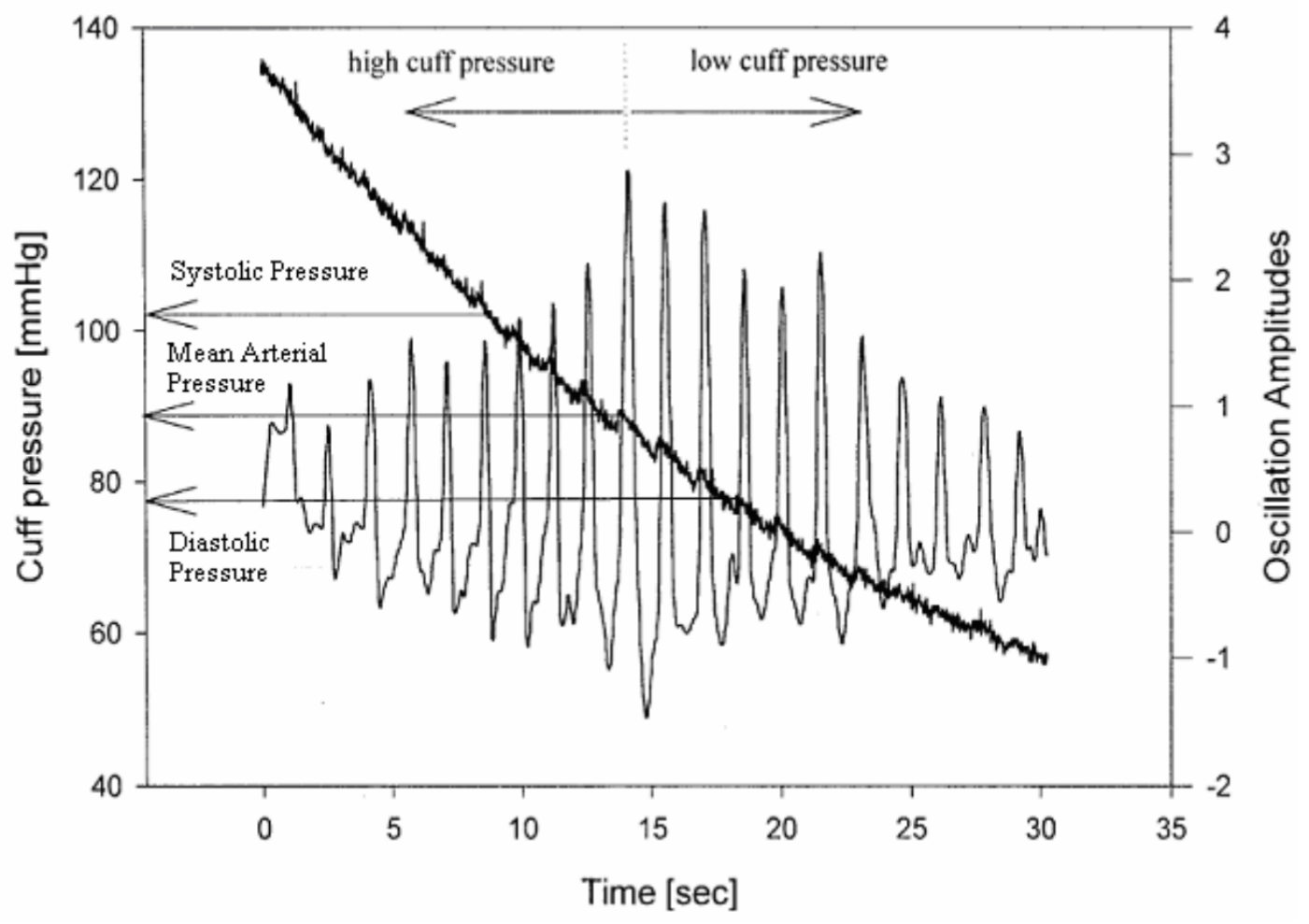

Fig. 1. Cuff pressure signal and oscillation waveform [8], [7]

There are two algorithms to determine the point of the systolic and the diastolic pressure, they called the heightbased and the slope-based method. The slope-based method fits a curve to the changes of cuff pressure. It specifies the inflection points of the curve as the points of systolic and diastolic pressures [2], [12], [7].

The height-based method has two predetermined ratios, one for systolic pressure and the other one for diastolic pressure. These two ratios are not necessarily the same. The pressure change at the point of mean arterial pressure is $100 \%$. The method finds the point where the pressure change corresponds to the given ratio. The point of systolic pressure is before, and the point of diastolic pressure is after the mean arterial pressure [2], [8], [6], [7].The ratios are not exactly determined. They depend on the realization of the oscillometric algorithm. Most researchers take the values between $40 \%$ and $60 \%$ [2], [7], [11], [3].

Oscillometric methods have been realized in many ways: prediction and smoothing algorithm, fuzzy logic, neural network, pattern recognition, mathematical modelling, etc. [7].

We based our method on the oscillometric method of [1].

\section{THE MICROCONTROLLER}

We chose the C8051F064 mixed-signal MCUs (Microcontroller unit) of Silicon Labories. These devices are equipped with $64 \mathrm{kB}$ Flash memory and $4 \mathrm{kB}$ data RAM.

The inflation and deflation of the cuff is controlled by the MCU. The cuff is inflated up to $180 \mathrm{mmHg}$ at the very first measurement and $20 \mathrm{mmHg}$ above the last measured systolic pressure later.

The MCU provides integer values. The sample rate is 200 $\mathrm{Hz}$. An integer value represents the cuff pressure value at a moment. After the inflation the first integer value is about 22860 , which is the cuff presure value $(180 \mathrm{mmHg})$ multiply 127.

The oscillometric algorithm works with the real time values. The MCU starts the blood pressure algorithm at the beginning of the deflation.

The data is stored permanently on a micro SD card. PC connection can be established easily through a USB cable, with which the PC has full access to the micro SD card.

\section{THE MAIN STEPS OF OUR ALGORITHM}

Input processing starts with the deflation of the cuff. First the algorithm perform a band-pass filter on the input, which means that the filter eliminates the deflation of the cuff. The result is an oscillogram, which shows only the cuff pressure changes caused the artery. Figure 2 shows the input values and the oscillogram after the deflation of the cuff.

Then, the algorithm finds the local extrema (minimum and maximum values) on the oscillogram. A histogram is constructed based on the extrema on the next way: a slope of the histogram maps the place (the time) of a local maximum to a difference between the maximum and the minimum pressure belonging to the same heart beat. 


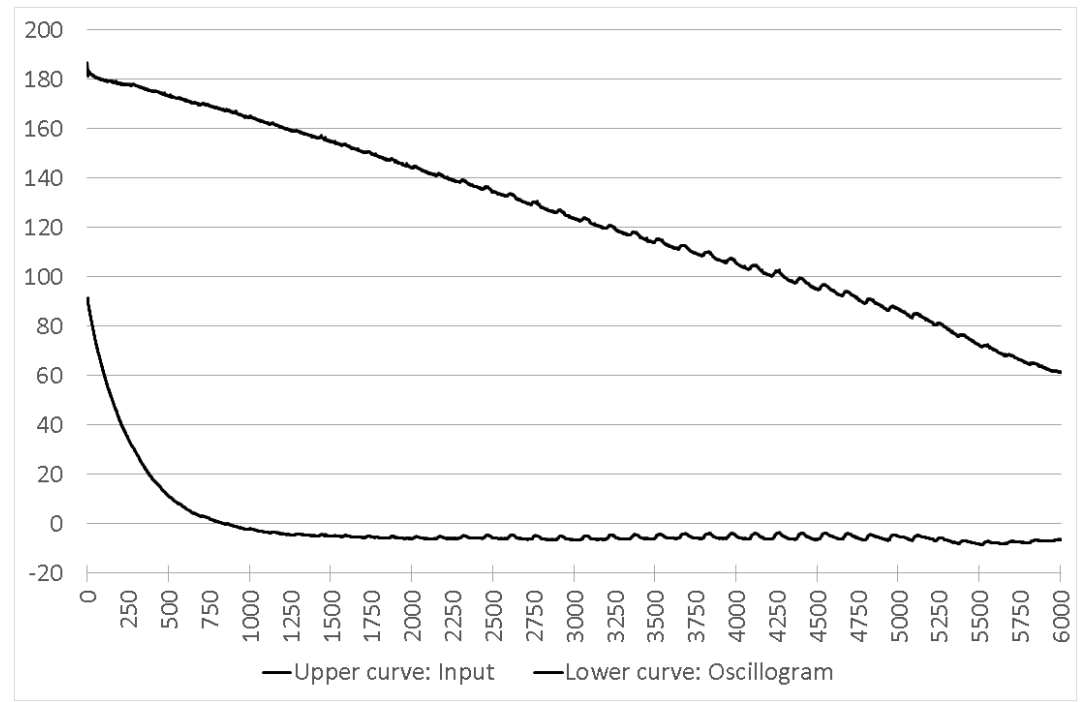

Fig. 2. Input signal and oscillogram of our algorithm

The histogram is used to compute the mean arterial pressure, the systolic pressure, the diastolic pressure and even an estimation for the heart rate. The MAP is the original cuff pressure value which belongs to the maximum value of the histogram. The algorithm uses the maximum value of the histogram to find the systolic and diastolic pressure values. There are two threshold values: $70 \%$ of the maximum value of the histogram for the systolic pressure and $65 \%$ for the diastolic pressure. In both case, the search begins at the maximum value of the histogram and terminates when adjacent values satisfies the following conditions: one of them is below, and the other one is above a threshold value. The search goes from the maximum value of the histogram to the left (to beginning of the input) in the systolic pressure case, and goes to the right (to end of the input) in the diastolic pressure case. Figure 3 shows the histogram, the maximum value of it (MAP), the thresholds, and the slopes represents the systole and the diastole.

The systolic pressure and the diastolic pressure are the original cuff pressure values which belongs to the place (time) of the proper slopes of the histogram.

A heart rate estimation can be made from the number of histogram values between the systolic pressure and the diastolic pressure values, and the elapsed time between them.

At the end of the algorithm we examine that the result is believable or not. If the systolic pressure is between 50 and $280 \mathrm{mmHg}$, the diastolic pressure between 40 and 140 and

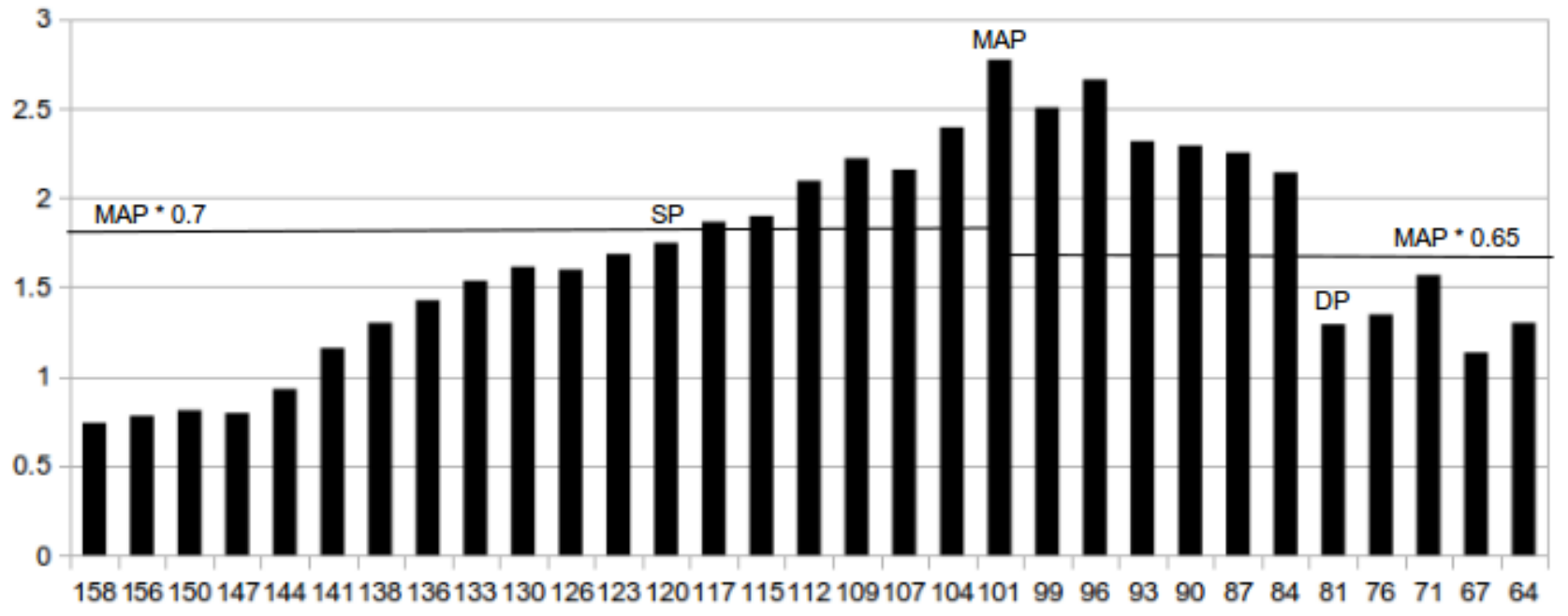

Fig. 3. Histogram 
the systolic pressure more then the diastolic pressure with $10 \mathrm{mmHg}$ the result is believable. Otherwise the result of algorithm is an error.

\section{Challenges of the MCU implementation}

A blood pressure measurement record typically consists of 6000-8000 of input values. An input value is a 16-bit unsigned integer, which represent a cuff pressure value. Clearly a complete record cannot be stored in the $4 \mathrm{kB}$ data RAM of the microcontroller, thus records are split into blocks containing 256 input values, then blocks are processed one-by-one. We have to remark that the last block might contain less than 256 input values.

The algorithm needs the time when produced the cuff pressure value. We can calculate this time based on its ordinal number in the block, the ordinal number of the block and the sample rate.

Extrema defined the following way: the $i^{\text {th }}$ input value is a local minimum (maximum) if it is less than (greater than) every $i \pm k^{\text {th }}$ input value, for $k=1, \ldots, \epsilon$, where $\epsilon$ is a predefined constant. Easy to see, that checking if the first value of a block is an extremum requiers the $(256-\epsilon)^{\text {th }}$ value of the previous block, also checking the last value of block requiers the $\epsilon^{\text {th }}$ value of the next block. Therefore, the last $2 \cdot \epsilon$ value of the recently processed block have to be kept in the memory, in order to find every extremum.

Band-pass filtering produces floating point values, but further use of the filtered values do not demand the accuracy of the floating point representation. To compute the $i^{\text {th }}$ filtered value the $(i-1)^{\text {th }}$ filtered value is required, so after the $i^{\text {th }}$ filtered value is computed, the $(i-1)^{\text {th }}$ filtered value is converted to signed integer and stored in that way.

Calculations required by curve-fitting techniques cannot be done real-time by the device. Therefore a histogram, which is produced from extrema, is used to find the systolic and diastolic pressure, and to make a heart-rate estimation. The data structure which represents the histogram in the memory of the MCU stores not only the slopes of the histogram, but the orginal values of the cuff pressure also.

\section{CONCLUSION}

We have introduced our oscillometric blood pressure measurement algorithm for microcontroller. Our device can transmit the measurement records made during a 24-hour interval to the BP Service application of the Cardiospy system of Labtech Ltd. Moreover, it displays informative systolic pressure, diastolic pressure and heart rate results to the patients. We also described the difficulties of the implementation of our oscillometric blood pressure measurement algorithm.

The algorithm works well under laboratory circumstances. Our goal is to meet the requirements specified by British Hypertension Society (BHS) [10] and European Society of Hypertension (ESH) [9] standards. Further testings required to see whether a parameter used by the algorithm needs a slightly change or not.

\section{ACKNOWLEDGMENT}

The authors thank Bela Kincs, Peter Toth and Bela Vamosi of Labtech Ltd for their valuable contributions.

\section{REFERENCES}

[1] AвоY, R., Method for blood pressure measurement from noninvasive oscillometric pressure signals. Technical report, Tiba Medical, Inc, (2011).

[2] Ball-llovera, A., Del Rey, R., Ruso, R., Ramos, J., Batista, O., AND NiUBO, I., An experience in implementing the oscillometric algorithm for the noninvasive determination of human blood pressure. Engineering in Medicine and Biology Society, Proceedings of the 25th Annual International Conference of the IEEE, Vol. 4 (2003), 3173-3175.

[3] Geddes, L. A., Handbook of Blood Pressure Measurement. Humana Press, 1st edition, (1991).

[4] Forouzanfar, M., Dajani, HR., Groza, VZ., Bolic, M., Rajan, S., BATKIN, I. Oscillometric Blood Pressure Estimation: Past, Present, and Future. IEEE Reviews in Biomedical Engineering, Vol. 8 (2015), 44-63.

[5] Labtech (2017). Labtech ltd. http://www.labtech.hu.

[6] LeE, J., Kim, J., AND Yoon, G., Digital envelope detector for blood pressure measurement using an oscillometric method. Journal Medical Engineering and Technology, Proceedings of the 23rd Annual International Conference of the IEEE, Vol. 1 (2001), 126-128.

[7] LIN, H.-C., Specialised non-invasive blood pressure measurement algorithm. Masters thesis, Auckland University of Technology, (2007).

[8] Lin, C.-T., LiU, S.-H., WANG, J.-J., And Wen, Z.-C., Reduction of interference in oscillometric arterial blood pressure measurement using fuzzy logic. IEEE Transactions on Biomedical Engineering, Vol. 50(4) (2003), 432-441.

[9] Obrien, E., Atkins, N., Stergiou, G., Karpettas, N., Parati, G., Asmar, R., Imai, Y., Wang, J., Mengden, T., and Shennan, A., European society of hypertension international protocol revision 2010 for the validation of blood pressure measuring devices in adults. Blood Pressure Monitoring, Vol. 15 (2010), 23-38.

[10] Obrien, E., Petrie, J., Littler, W., De Swiet, M., Padfield, P. L., Altman, D. G., Bland, M., Coats, A., And Atkins, N., The british hypertension society protocol for the evaluation of blood pressure measuring devices. Journal of Hypertension, Vol. 11 (1993), 43-62.

[11] SAPINSKI, A., Standard algorithm of blood-pressure measurement by the oscillometric method. Medical and Biological Engineering and Computing, Vol. 30 (1992), 671.

[12] Sapinski, A., Theoretical basis for proposed standard algorithm of blood pressure measurement by the sphygmooscillographic method. Journal Of Clinical Engineering, Vol. 22 (1997), 171-174.

[13] VÁGner, A., VÁmosi, B., AND JuHÁsz, I., Visualization and Off-line Processing of Blood Pressure Signals. Proceedings of the International Conference on Health Informatics (7th International Conference on Health Informatics) (HEALTHINF 2014), (2014), 393-398.

[14] WANG, J.-J., LIN, C.-T., LIU, S.-H., AND WEN, Z.-C., Model-based synthetic fuzzy logic controller for indirect blood pressure measurement. IEEE Transactions on Systems, Man, and Cybernetics, Part B: Cybernetics archive, Vol. 32(3) (2002), 306-315. 